\title{
Positive Solutions to a Boundary Value Problem for the Beam Equation
}

\author{
Bo Yang
}

\begin{abstract}
We consider a two-point boundary value problem for the beam equation, in which the boundary conditions mean that the beam is simply supported at both ends. Some a priori estimates to positive solutions for the problem are obtained. Existence and nonexistence results for positive solutions of the problem are established.
\end{abstract}

Keywords. Positive solutions, higher order boundary value problem, fixed point

Mathematics Subject Classification (2000). Primary 34B18, secondary 34B15

\section{Introduction}

We consider the fourth order boundary value problem

$$
\begin{aligned}
& u^{\prime \prime \prime \prime}(t)=g(t) f(u(t)), \quad 0 \leq t \leq 1 \\
& u(0)=u^{\prime \prime}(0)=u^{\prime \prime}(1)=u(1)=0
\end{aligned}
$$

The equation (1.1) and the boundary conditions (1.2) arise from the study of elasticity and have definite physical meanings. The equation (1.1) describes the deflection of a beam under a certain force. The boundary conditions (1.2) mean that the beam is simply supported (also called fulcrum supported) at both ends $t=0$ and $t=1$.

The beam equation has been studied by many authors under various boundary conditions and by different approaches. For example, the problem (1.1)(1.2) was investigated by Agarwal [1], Bai and Wang [2], Ma and Wang [12], and Graef and Yang [6]. For some other results on boundary value problems of the beam equation, we refer the reader to the papers of Davis and Henderson [3], Elgindi and Guan [4], Eloe, Henderson, and Kosmatov [5], Gupta [7], Kosmatov [8], Liu and Ge [10], Ma [11], Yang [13], and Yao [14].

The main purpose of this paper is to study the existence and nonexistence of positive solutions to the problem (1.1)-(1.2). By positive solution, we mean

Bo Yang: Department of Mathematics, Kennesaw State University, Kennesaw, GA 30144, USA; byang@kennesaw.edu 
a solution $u(t)$ such that $u(t)>0$ for $t \in(0,1)$. Throughout the paper we assume that

(H1) $f:[0, \infty) \rightarrow[0, \infty)$ and $g:[0,1] \rightarrow[0, \infty)$ are continuous functions, and $g(t) \not \equiv 0$ on $[0,1]$.

We will use the Guo-Krasnosel'skii fixed point theorem, stated below, to prove some of the results. First we recall that a nonempty subset $P$ of a real Banach space $X$ is called a cone if $P$ is closed, convex, and satisfies the following conditions:

(i) if $x \in P,-x \in P$, then $x=0$;

(ii) if $\lambda>0$ is a real number, $x \in P$, then $\lambda x \in P$.

Theorem $1.1([9])$. Let $(X,\|\cdot\|)$ be a Banach space over the reals, and let $P \subset X$ be a cone in $X$. Assume that $\Omega_{1}$ and $\Omega_{2}$ are bounded open subsets of $X$ with $0 \in \Omega_{1} \subset \overline{\Omega_{1}} \subset \Omega_{2}$, and let $L: P \cap\left(\overline{\Omega_{2}}-\Omega_{1}\right) \rightarrow P$ be a completely continuous operator such that, either

(K1) $\|L u\| \leq\|u\|$ if $u \in P \cap \partial \Omega_{1}$, and $\|L u\| \geq\|u\|$ if $u \in P \cap \partial \Omega_{2}$; or

(K2) $\|L u\| \geq\|u\|$ if $u \in P \cap \partial \Omega_{1}$, and $\|L u\| \leq\|u\|$ if $u \in P \cap \partial \Omega_{2}$.

Then $L$ has a fixed point in $P \cap\left(\overline{\Omega_{2}}-\Omega_{1}\right)$.

Before the Guo-Krasnosel'skii fixed point theorem can be used to obtain any existence result, we have to find some nice estimates to the positive solutions to the problem (1.1)-(1.2) first. Better estimates result in sharper existence and nonexistence conditions. Since the boundary conditions (1.2) are of the Lidstone type, the problem (1.1)-(1.2) has no monotonic solutions. This makes the task of finding estimates difficult, because it is not known where a solution to problem (1.1)-(1.2) achieves its maximum. To the author's knowledge, no satisfactory estimates have been obtained in the literature. One of the purposes of this paper is to establish some new a priori estimates to positive solutions of the problem (1.1)-(1.2), which improve the ones used in the literature. These a priori estimates are essential to the main results of this paper. It is based on these estimates that we can define an appropriate cone, on which Theorem 1.1 will be applied.

The Green's function $G:[0,1] \times[0,1] \rightarrow[0, \infty)$ for problem $(1.1)-(1.2)$ is given by

$$
G(t, s)= \begin{cases}\frac{1}{6} t(1-s)\left(2 s-s^{2}-t^{2}\right), & \text { if } 0 \leq t \leq s \leq 1 \\ \frac{1}{6} s(1-t)\left(2 t-s^{2}-t^{2}\right), & \text { if } 0 \leq s \leq t \leq 1\end{cases}
$$

Then problem (1.1)-(1.2) is equivalent to the integral equation

$$
u(t)=\int_{0}^{1} G(t, s) g(s) f(u(s)) d s, \quad 0 \leq t \leq 1 .
$$


It's easy to verify that $G(t, s)>0$ if $t, s \in(0,1)$. Throughout this paper, we let

$$
\begin{aligned}
F_{0} & =\limsup _{x \rightarrow 0^{+}} \frac{f(x)}{x}, & f_{0} & =\liminf _{x \rightarrow 0^{+}} \frac{f(x)}{x} \\
F_{\infty} & =\limsup _{x \rightarrow+\infty} \frac{f(x)}{x}, & f_{\infty} & =\liminf _{x \rightarrow+\infty} \frac{f(x)}{x},
\end{aligned}
$$

and let $X=C[0,1]$ with the supremum norm $\|u\|=\max _{0 \leq t \leq 1}|u(t)|$. Obviously, $X$ is a Banach space.

This paper is organized as follows. In Section 2, new estimates to the positive solutions to problem (1.1)-(1.2) are obtained through detailed analysis. In Sections 3 and 4, we establish some existence and nonexistence results for positive solutions to this problem. An example is given at the end of the paper to illustrate the main results.

\section{Estimates to positive solutions}

In this section, we present both upper and lower estimates to positive solutions for the problem (1.1)-(1.2).

Lemma 2.1. Let $u \in C^{2}[0,1]$. Suppose that $u(0)=u(1)=0$ and $u(r)=0$ for some $r \in(0,1)$. If $u^{\prime \prime}(t) \leq 0$ on $[0,1]$, then $u(t) \equiv 0$ on $[0,1]$.

Lemma 2.2. If $u \in C^{4}[0,1]$ satisfies (1.2) and

$$
u^{\prime \prime \prime \prime}(t) \geq 0 \quad \text { for } 0 \leq t \leq 1,
$$

then $u(t) \geq 0$ and $u^{\prime \prime}(t) \leq 0$ for $0 \leq t \leq 1$. If, in addition, $u(r)>0$ for some $r \in(0,1)$, then $u(t)>0$ and $u^{\prime \prime}(t)<0$ for $0<t<1$.

The proofs of Lemmas 2.1 and 2.2 are straightforward and left to the reader. One implication of the two lemmas is that if $u \in C^{4}[0,1]$ satisfies (1.2) and (2.1), then either $u(t) \equiv 0$ on $[0,1]$, or $u(t)>0$ for $0<t<1$. Another implication of the two lemmas is that, under condition (H1), every solution of the problem (1.1)-(1.2) is a nonnegative solution.

Lemma 2.3. Suppose that $u \in C^{4}[0,1]$ satisfies (1.2) and (2.1), and such that

$$
u(t)>0 \text { for } 0<t<1 \text {. }
$$

Then there exists a unique $c \in(0,1)$ such that $u^{\prime}(c)=0$. In this case, $u(c)=\|u\|$.

Proof. Since $u(0)=u(1)=0$, there exists $c \in(0,1)$ such that $u^{\prime}(c)=0$. Since $u^{\prime \prime}(t)<0$ on $[0,1]$, this $c$ must be unique, and

$$
u^{\prime}(t)>0 \quad \text { for } 0 \leq t<c, \quad u^{\prime}(t)<0 \quad \text { for } c<t \leq 1 .
$$

It follows that $u(c)=\|u\|$. The proof of the lemma is complete. 
Lemma 2.4. Suppose $u \in C^{4}[0,1]$ satisfies (1.2), (2.1), and (2.2). Let $c$ be the unique zero of $u^{\prime}$ in $[0,1]$, and let $p(t)=-u^{\prime \prime}(t), 0 \leq t \leq 1$. Then $p(0)=p(1)=0$, $p(c)>0, p(t)$ is concave downward on $[0,1]$,

$$
\begin{array}{rlr}
p(t) & \geq \frac{t}{c} p(c) & \text { on }[0, c] \\
p(t) & \leq \frac{t}{c} p(c) & \text { on }[c, 1] \\
\int_{0}^{c} t p(t) d t & =\int_{c}^{1}(1-t) p(t) d t .
\end{array}
$$

Proof. It follows from (1.2) that $p(0)=p(1)=0$. Note that $(2.1)$ implies that $p(t)$ is concave downward. By Lemma 2.2, we have $p(c)>0$. Now (2.4) and (2.5) follow immediately from the concavity of $p(t)$.

It is easy to see that $\int_{0}^{c} t p(t) d t=u(c)=\int_{c}^{1}(1-t) p(t) d t$, and (2.6) follows immediately. The proof is complete.

Throughout the remainder of the paper, we define the constants

$$
Q=\frac{9}{16} \sqrt{3}, \quad c_{1}=1-\frac{1}{3} \sqrt{3}, \quad c_{2}=\frac{1}{3} \sqrt{3},
$$

and the functions $a:[0,1] \rightarrow[0,1]$ and $b:[0,1] \rightarrow[0,1]$ by

$$
\begin{aligned}
& a(t)= \begin{cases}\frac{3 \sqrt{3}}{2}\left(t-t^{3}\right), & \text { if } 0 \leq t \leq \frac{1}{2} \\
\frac{3 \sqrt{3}}{2}\left(t^{3}-3 t^{2}+2 t\right), & \text { if } \frac{1}{2} \leq t \leq 1\end{cases} \\
& b(t)= \begin{cases}\frac{3 \sqrt{3}}{2}\left(t^{3}-3 t^{2}+2 t\right), & \text { if } 0 \leq t \leq c_{1} \\
1, & \text { if } c_{1} \leq t \leq c_{2} \\
\frac{3 \sqrt{3}}{2}\left(t-t^{3}\right), & \text { if } c_{2} \leq t \leq 1\end{cases}
\end{aligned}
$$

The functions $a(t)$ and $b(t)$ will be used to estimate the positive solutions of the problem $(1.1)-(1.2)$. It is easy to verify that $Q=a\left(\frac{1}{2}\right)$, both $a(t)$ and $b(t)$ are continuous functions, $a(t)=a(1-t), b(t)=b(1-t)$, and $b(t) \geq a(t) \geq$ $2 Q \min \{t, 1-t\}$ for $0 \leq t \leq 1$.

Lemma 2.5. Suppose $u \in C^{4}[0,1]$ satisfies (1.2), (2.1), and (2.2). Let $c$ be the unique zero of $u^{\prime}$ in $(0,1)$. Then $c_{1}<c<c_{2}$, and

$$
u(t) \geq \frac{3}{2} \sqrt{3}\left(t-t^{3}\right)\|u\| \quad \text { for } 0 \leq t \leq c .
$$

Proof. Let $p(t)=-u^{\prime \prime}(t), 0 \leq t \leq 1$. In view of (2.4), we have

$$
\int_{0}^{c} t p(t) d t \geq \int_{0}^{c} \frac{t^{2}}{c} p(c) d t=\frac{c^{2}}{3} p(c) .
$$


Note that $p(1)=0$ and $p(c)>0$. Since $p(t)$ is continuous, there exists $\delta \in(c, 1)$ such that $p(t)<\frac{t}{c} p(c)$ for $\delta<t<1$, which together with (2.5) implies that

$$
\int_{c}^{1}(1-t) p(t) d t<\int_{c}^{1}(1-t) \frac{t}{c} p(c) d t=\left(\frac{1}{6 c}-\frac{c}{2}+\frac{c^{2}}{3}\right) p(c) .
$$

Substituting (2.8) and (2.9) into (2.6), we arrive at $\frac{c^{2}}{3} p(c)<\left(\frac{1}{6 c}-\frac{c}{2}+\frac{c^{2}}{3}\right) p(c)$, which implies that $c<\frac{\sqrt{3}}{3}=c_{2}$.

To show that $c>c_{1}$, we let $v(t)=u(1-t), 0 \leq t \leq 1$. It is obvious that $v(0)=v^{\prime \prime}(0)=v^{\prime \prime}(1)=v(1)=0, v(t)>0$ for $0<t<1$, and $v^{\prime \prime \prime \prime}(t)=$ $u^{\prime \prime \prime \prime}(1-t) \geq 0,0 \leq t \leq 1$. Since $c$ is the unique zero of $u^{\prime}$ in $(0,1), 1-c$ is the unique zero of $v^{\prime}$ in $(0,1)$. From the early portion of the proof, we see that $1-c<\frac{\sqrt{3}}{3}$. It follows immediately that $c>1-\frac{\sqrt{3}}{3}=c_{1}$.

If we define

$$
h(t)=u(t)-\frac{3}{2} \sqrt{3}\left(t-t^{3}\right) u(c), \quad 0 \leq t \leq c,
$$

then

$$
\begin{aligned}
h^{\prime}(t) & =u^{\prime}(t)-\frac{3}{2} \sqrt{3}\left(1-3 t^{2}\right) u(c) \\
h^{\prime \prime}(t) & =u^{\prime \prime}(t)+9 \sqrt{3} t u(c) \\
h^{\prime \prime \prime \prime}(t) & =u^{\prime \prime \prime \prime}(t) \geq 0, \quad 0 \leq t \leq c .
\end{aligned}
$$

To prove (2.7), it suffices to show that $h(t) \geq 0$ for $0 \leq t \leq c$. Note that (2.13) implies that $h^{\prime \prime}$ is concave upward. We see from (2.10) and (2.12) that $h(0)=h^{\prime \prime}(0)=0$. For convenience, we define an auxiliary function

$$
\varphi(t)=\frac{3}{2} \sqrt{3}\left(t-t^{3}\right), \quad 0 \leq t \leq 1 .
$$

It's easy to verify that $\varphi\left(c_{2}\right)=1, \varphi^{\prime}\left(c_{2}\right)=0, \varphi(t)$ is strictly increasing on $\left[0, c_{2}\right]$, and $\varphi^{\prime}(t)$ is strictly decreasing on $\left[0, c_{2}\right]$. Because $c<c_{2}$, we have $h(c)=u(c)-$ $\varphi(c) u(c)>u(c)-\varphi\left(c_{2}\right) u(c)=0$ and $h^{\prime}(c)=-\varphi^{\prime}(c) u(c)<-\varphi^{\prime}\left(c_{2}\right) u(c)=0$. By the Mean Value Theorem, because $h(0)=0<h(c)$, there exists $r_{1} \in(0, c)$ such that $h^{\prime}\left(r_{1}\right)>0$. Because $h^{\prime}\left(r_{1}\right)>0>h^{\prime}(c)$, there exists $r_{2} \in\left(r_{1}, c\right)$ such that $h^{\prime \prime}\left(r_{2}\right)<0$. At this point, there are two possible cases:

Case I: $h^{\prime \prime}(c) \leq 0$. In this case, because $h^{\prime \prime}(0)=0$ and because $h^{\prime \prime}$ is concave upward, we have $h^{\prime \prime}(t) \leq 0$ for $0 \leq t \leq c$. Therefore $h(t)$ is concave downward. Because $h(0)=0$ and $h(c)>0$, we have $h(t) \geq 0$ for $0 \leq t \leq c$.

Case II: $\quad h^{\prime \prime}(c)>0$. In this case, because $h^{\prime \prime}(0)=0, h^{\prime \prime}\left(r_{2}\right)<0$, and $h^{\prime \prime}$ is concave upward, there exists $r_{3} \in\left(r_{2}, c\right)$ such that $h^{\prime \prime}(t) \leq 0$ for $t \in\left[0, r_{3}\right]$, and $h^{\prime \prime}(t) \geq 0$ for $t \in\left[r_{3}, c\right]$. Since $h(c)>0$ and $h^{\prime}(c)<0$, and $h(t)$ is concave upward on $\left[r_{3}, c\right]$, we have $h(t)>0$ for $r_{3} \leq t \leq c$. Because $h(0)=0, h\left(r_{3}\right)>0$, and $h(t)$ is concave downward on $\left[0, r_{3}\right]$, we have $h(t)>0$ for $0<t \leq r_{3}$.

In either case, we have $h(t) \geq 0$ for $t \in[0, c]$. The proof is complete. 
Lemma 2.6. If $u \in C^{4}[0,1]$ satisfies (1.2), (2.1), and (2.2), then

$$
u(t) \leq b(t)\|u\| \quad \text { for } 0 \leq t \leq c_{1} .
$$

Proof. Let $c$ be the unique zero of $u^{\prime}$ in $(0,1)$. If we define

$$
h(t)=b(t) u(c)-u(t)=\frac{3}{2} \sqrt{3}\left(t^{3}-3 t^{2}+2 t\right) u(c)-u(t), \quad 0 \leq t \leq c_{1},
$$

then

$$
\begin{aligned}
h^{\prime}(t) & =\frac{3}{2} \sqrt{3}\left(3 t^{2}-6 t+2\right) u(c)-u^{\prime}(t) \\
h^{\prime \prime}(t) & =9 \sqrt{3}(t-1) u(c)-u^{\prime \prime}(t) \\
h^{\prime \prime \prime \prime}(t) & =-u^{\prime \prime \prime \prime}(t) \leq 0, \quad 0 \leq t \leq c_{1} .
\end{aligned}
$$

From (2.14) and (2.16) we see that $h(0)=0$ and $h^{\prime \prime}(0)<0$. Since $0<c_{1}<c$, (2.3) implies that $u^{\prime}\left(c_{1}\right)>0$ and $u\left(c_{1}\right)<u(c)$. Therefore, $h\left(c_{1}\right)=\frac{3}{2} \sqrt{3}\left(c_{1}^{3}-3 c_{1}^{2}+\right.$ $\left.2 c_{1}\right) u(c)-u\left(c_{1}\right)=u(c)-u\left(c_{1}\right)>0$, and $h^{\prime}\left(c_{1}\right)=\frac{3}{2} \sqrt{3}\left(3 c_{1}^{2}-6 c_{1}+2\right) u(c)-u^{\prime}\left(c_{1}\right)=$ $-u^{\prime}\left(c_{1}\right)<0$.

Claim. If $h^{\prime \prime}\left(c_{1}\right) \leq 0$, then $h^{\prime \prime}(t) \leq 0$ for $t \in\left[0, c_{1}\right]$.

Proof of the Claim. Suppose that $h^{\prime \prime}\left(c_{1}\right) \leq 0$. Assume the contrary that there exists $\beta \in\left(0, c_{1}\right)$ such that $h^{\prime \prime}(\beta)>0$. Let $p(t)=-u^{\prime \prime}(t), 0 \leq t \leq 1$. Note that $h^{\prime \prime}\left(c_{1}\right) \leq 0$ implies that $p\left(c_{1}\right) \leq 9 u(c)$; and $h^{\prime \prime}(\beta)>0$ implies that $p(\beta)>$ $9 \sqrt{3}(1-\beta) u(c)$. Because $p(t)$ is concave downward, we have

$$
p(t) \leq p\left(c_{1}\right)+\frac{p\left(c_{1}\right)-p(\beta)}{c_{1}-\beta}\left(t-c_{1}\right) \quad \text { for } t>c_{1} .
$$

Therefore,

$$
\frac{p(t)}{u(c)}<9+\frac{9-9 \sqrt{3}(1-\beta)}{c_{1}-\beta}\left(t-c_{1}\right)=9-9 \sqrt{3}\left(t-c_{1}\right), \quad t>c_{1} .
$$

It follows that $p(1)<u(c)\left(9-9 \sqrt{3}\left(1-c_{1}\right)\right)=0$, which contradicts the fact that $p(1)=0$. The proof of the Claim is complete.

At this point there are two possible cases:

Case I: $h^{\prime \prime}\left(c_{1}\right) \leq 0$. In this case, by the claim, we have $h^{\prime \prime}(t) \leq 0$ for $t \in\left[0, c_{1}\right]$. Therefore $h(t)$ is concave downward. Because $h(0)=0$ and $h\left(c_{1}\right)>0$, we have $h(t) \geq 0$ for $0 \leq t \leq c_{1}$.

Case II: $h^{\prime \prime}\left(c_{1}\right)>0$. In this case, because $h^{\prime \prime}$ is concave downward, and $h^{\prime \prime}(0)<0$, there exists $t_{0} \in\left(0, c_{1}\right)$ such that $h^{\prime \prime}(t) \leq 0$ on $\left[0, t_{0}\right], h^{\prime \prime}(t) \geq 0$ on $\left[t_{0}, c_{1}\right]$. Because $h\left(c_{1}\right)>0, h^{\prime}\left(c_{1}\right)<0$, and $h(t)$ is concave upward on $\left[t_{0}, c_{1}\right]$, we have $h(t)>0$ for $t_{0} \leq t \leq c_{1}$. Because $h(t)$ is concave downward on $\left[0, t_{0}\right]$, $h(0)=0$, and $h\left(t_{0}\right)>0$, we have $h(t) \geq 0$ for $0 \leq t \leq t_{0}$.

In either case, we have $h(t) \geq 0$ for $t \in\left[0, c_{1}\right]$. The proof is complete. 
Theorem 2.7. If $u \in C^{4}[0,1]$ satisfies (1.2), (2.1), and (2.2), then we have

$$
a(t)\|u\| \leq u(t) \leq b(t)\|u\| \quad \text { for } 0 \leq t \leq 1 .
$$

Proof. Let $c$ be the unique zero of $u^{\prime}$ in $(0,1)$. First, we note that

$$
a(t)=\min \left\{\frac{3}{2} \sqrt{3}\left(t-t^{3}\right), \frac{3}{2} \sqrt{3}\left(t^{3}-3 t^{2}+2 t\right)\right\}, \quad 0 \leq t \leq 1 .
$$

From Lemma 2.5 we see that $u(t) \geq a(t) u(c)$ for $0 \leq t \leq c$. If we let $v(t)=$ $u(1-t), 0 \leq t \leq 1$, then $1-c$ is the unique zero of $v^{\prime}$ in $[0,1]$. By applying Lemma 2.5 to $v(t)$, we get $v(t) \geq a(t) v(1-c)$ for $0 \leq t \leq 1-c$, which implies that $u(t) \geq a(t) u(c)$ for $c \leq t \leq 1$. Thus we proved the left half of (2.18).

From Lemma 2.6 we see that $u(t) \leq b(t) u(c)$ for $0 \leq t \leq c_{1}$. By applying Lemma 2.6 to $v(t)=u(1-t)$ we get $u(t) \leq b(t) u(c)$ for $c_{2} \leq t \leq 1$. And it is obvious that $u(t) \leq\|u\|=b(t)\|u\|$ for $c_{1} \leq t \leq c_{2}$. Thus we proved the right half of (2.18). The proof is complete.

The next theorem is a direct consequence of Theorem 2.7.

Theorem 2.8. If $u \in C^{4}[0,1]$ satisfies (1.2), (2.1), (2.2), then $u\left(\frac{1}{2}\right) \geq Q\|u\|$, and

$$
a(t)\|u\| \leq u(t) \leq Q^{-1} u\left(\frac{1}{2}\right) b(t) \quad \text { on }[0,1] .
$$

In particular, if $u(t)$ is a solution to the problem (1.1)-(1.2), then $u\left(\frac{1}{2}\right) \geq Q\|u\|$ and $u(t)$ satisfies $(2.19)$.

Remark 2.9. Theorem 2.8 provides not only an upper estimate but also a lower estimate to positive solutions for the problem (1.1)-(1.2). These estimates are finer than the one used in [12], namely, if $u(t)$ is a nonnegative solution to the problem (1.1)-(1.2), then

$$
u(t) \geq \frac{1}{4}\|u\| \quad \text { for } \frac{1}{4} \leq t \leq \frac{3}{4} .
$$

The estimate (2.20) was used to define the positive cone $P_{1}$ in $[12$, p. 230]. It was also used to define the positive cone $P$ in the paper [2, p. 358].

\section{Existence results}

We define the constants $A$ and $B$ by

$$
A=\int_{0}^{1} G\left(\frac{1}{2}, s\right) g(s) a(s) d s, \quad B=\int_{0}^{1} G\left(\frac{1}{2}, s\right) g(s) b(s) d s .
$$

Also we define a subset $P$ of $X$ by

$$
P=\left\{v \in X \mid a(t)\|v\| \leq v(t) \leq Q^{-1} v\left(\frac{1}{2}\right) b(t) \text { for } 0 \leq t \leq 1\right\}
$$


and the operator $T: P \rightarrow X$ by

$$
(T u)(t)=\int_{0}^{1} G(t, s) g(s) f(u(s)) d s, \quad 0 \leq t \leq 1, \forall u \in X .
$$

Clearly, $P$ is a positive cone in $X$, and $T: P \rightarrow X$ is a completely continuous operator. It is easily seen from the definition of $P$ that if $v \in P$, then $\|v\| \leq$ $Q^{-1} v\left(\frac{1}{2}\right)$. Note that if $u \in P$, then $u(t) \geq 0$ for $0 \leq t \leq 1$. It follows from Theorem 2.8 that if $u \in C^{4}[0,1]$ satisfies (1.2), (2.1), and (2.2), then $u \in P$. In particular, if $u(t)$ is a solution to the problem (1.1)-(1.2), then $u \in P$. In a similar fashion to Theorem 2.8, we can show that $T(P) \subset P$.

Now we are ready to prove the existence results.

Theorem 3.1. Suppose that (H1) holds. If $Q^{-1} B F_{0}<1<A f_{\infty}$, then the problem (1.1)-(1.2) has at least one positive solution.

Proof. Though the proof is somewhat similar to the ones in [13], it is included here for the purpose of completeness. First, we choose $\varepsilon>0$ such that $Q^{-1}\left(F_{0}+\right.$ $\varepsilon) B \leq 1$. There exists $H_{1}>0$ such that $f(x) \leq\left(F_{0}+\varepsilon\right) x$ for $0<x \leq H_{1}$. For each $u \in P$ with $\|u\|=H_{1}$, we have

$$
\begin{aligned}
\|T u\| \leq Q^{-1}(T u)\left(\frac{1}{2}\right) & =Q^{-1} \int_{0}^{1} G\left(\frac{1}{2}, s\right) g(s) f(u(s)) d s \\
& \leq Q^{-1}\left(F_{0}+\varepsilon\right)\|u\| \int_{0}^{1} G\left(\frac{1}{2}, s\right) g(s) b(s) d s \\
& =Q^{-1}\left(F_{0}+\varepsilon\right)\|u\| B
\end{aligned}
$$

which means $\|T u\| \leq\|u\|$. Thus, if we let $\Omega_{1}=\left\{u \in X \mid\|u\|<H_{1}\right\}$, then $\|T u\| \leq\|u\|$ for $u \in P \cap \partial \Omega_{1}$. To construct $\Omega_{2}$, we choose $\tau \in\left(0, \frac{1}{4}\right)$ and $\delta>0$ such that

$$
\int_{\tau}^{1-\tau} G\left(\frac{1}{2}, s\right) g(s) a(s) d s \cdot\left(f_{\infty}-\delta\right)>1 .
$$

There exists $H_{3}>0$ such that $f(x) \geq\left(f_{\infty}-\delta\right) x$ for $x \geq H_{3}$. Let $H_{2}=$ $\max \left\{\frac{H_{3}}{\tau}, 2 H_{1}\right\}$. If $u \in P$ with $\|u\|=H_{2}$, then for each $t \in[\tau, 1-\tau]$, we have

$$
u(t) \geq H_{2} a(t) \geq H_{2}(2 Q) \min \{t, 1-t\} \geq H_{2} \min \{t, 1-t\} \geq H_{2} \tau \geq H_{3} .
$$

Therefore, for each $u \in P$ with $\|u\|=H_{2}$, we have

$$
\begin{aligned}
(T u)\left(\frac{1}{2}\right) & =\int_{0}^{1} G\left(\frac{1}{2}, s\right) g(s) f(u(s)) d s \\
& \geq \int_{\tau}^{1-\tau} G\left(\frac{1}{2}, s\right) g(s) f(u(s)) d s \\
& \geq \int_{\tau}^{1-\tau} G\left(\frac{1}{2}, s\right) g(s) a(s) d s \cdot\left(f_{\infty}-\delta\right)\|u\|
\end{aligned}
$$


which means $\|T u\| \geq\|u\|$. Thus, if we let $\Omega_{2}=\left\{u \in X \mid\|u\|<H_{2}\right\}$, then $\overline{\Omega_{1}} \subset \Omega_{2}$, and $\|T u\| \geq\|u\|$ for $u \in P \cap \partial \Omega_{2}$. The conditions in part (K1) of Theorem 1.1 are then satisfied, so there exists a fixed point $u$ of $T$ in $P$ such that $H_{1} \leq\|u\| \leq H_{2}$. The proof is complete.

Theorem 3.2. Suppose that (H1) holds. If $Q^{-1} B F_{\infty}<1<A f_{0}$, then the problem (1.1)-(1.2) has at least one positive solution.

The proof of Theorem 3.2 is quite similar to that of Theorem 3.1 and therefore omitted.

\section{Nonexistence results and example}

In this section, we present two nonexistence results for positive solutions to the problem (1.1)-(1.2).

Theorem 4.1. Suppose that (H1) holds. If $Q^{-1} B f(x)<x$ for all $x>0$, then the problem (1.1)-(1.2) has no positive solutions.

Proof. Assume the contrary that $u(t)$ is a positive solution of the problem (1.1)(1.2). Then $u \in P, u(t)>0$ for $0<t<1$, and

$u\left(\frac{1}{2}\right)=\int_{0}^{1} G\left(\frac{1}{2}, s\right) g(s) f(u(s)) d s<Q B^{-1} \int_{0}^{1} G\left(\frac{1}{2}, s\right) g(s) u(s) d s \leq Q\|u\| \leq u\left(\frac{1}{2}\right)$, which is a contradiction. The proof is complete.

Theorem 4.2. Suppose that (H1) holds. If $A f(x)>x$ for all $x>0$, then the problem (1.1)-(1.2) has no positive solutions.

Proof. Assume the contrary that $u(t)$ is a positive solution of the problem (1.1)(1.2). Then $u \in P, u(t)>0$ for $0<t<1$, and

$$
u\left(\frac{1}{2}\right)=\int_{0}^{1} G\left(\frac{1}{2}, s\right) g(s) f(u(s)) d s>A^{-1} \int_{0}^{1} G\left(\frac{1}{2}, s\right) g(s) a(s) d s \cdot\|u\|=\|u\|,
$$

which is a contradiction. The proof is complete.

Example 4.3. Consider the boundary value problem

$$
\begin{aligned}
& u^{\prime \prime \prime \prime}(t)=g(t) f(u(t)), \quad 0 \leq t \leq 1 \\
& u(0)=u^{\prime \prime}(0)=u^{\prime \prime}(1)=u(1)=0
\end{aligned}
$$

where $g(t)=4 t-3 t^{2}$ for $0 \leq t \leq 1, f(u)=\lambda \frac{u(1+2 u)}{1+u}, u \geq 0$, and $\lambda>0$ is a parameter. Obviously $F_{0}=f_{0}=\lambda, F_{\infty}=f_{\infty}=2 \lambda$. And it is easy to see that $\lambda x<f(x)<2 \lambda x$ for $x>0$. Calculations indicate that $A=\frac{1067}{172032} \sqrt{3}$ and $B=\frac{23}{1440} \sqrt{3}-\frac{9121}{622080}$. By Theorem 3.1, if 46.542981 $\approx \frac{1}{2 A}<\lambda<\frac{Q}{B} \approx 74.92952$, then the problem (4.1)-(4.2) has at least one positive solution. By Theorems 4.1 and 4.2, we have that if either $\lambda \leq \frac{Q}{2 B} \approx 37.46476$ or $\lambda \geq \frac{1}{A} \approx 93.08596$, then the problem (4.1)-(4.2) has no positive solutions. 
Acknowledgement. The author is grateful to the anonymous referees for their careful reading of the manuscript and valuable suggestions.

\section{References}

[1] Agarwal, R. P., On fourth-order boundary value problems arising in beam analysis. Diff. Integral Equations 2 (1989), 91 - 110.

[2] Bai, Z. and Wang, H., On positive solutions of some nonlinear fourth-order beam equations. J. Math. Anal. Appl. 270 (2002)(2), $357-368$.

[3] Davis, J. and Henderson, J., Uniqueness implies existence for fourth-order Lidstone boundary value problems, Panamer. Math. J. 8 (1998)(4), 23 - 35.

[4] Elgindi, M. B. M. and Guan, Z., On the global solvability of a class of fourthorder nonlinear boundary value problems. Internat. J. Math. Math. Sci. 20 (1997)(2), $257-262$.

[5] Eloe, P. W., Henderson, J. L. and Kosmatov, N., Countable positive solutions of a conjugate type boundary value problem. Comm. Appl. Nonlinear Anal. 7 (2000), $47-55$.

[6] Graef, J. R. and Yang, B., On a nonlinear boundary value problem for fourth order equations. Appl. Anal. 72 (1999)(3-4), $439-448$.

[7] Gupta, C. P., Existence and uniqueness theorems for the bending of an elastic beam equation. Appl. Anal. 26 (1988)(4), 289 - 304.

[8] Kosmatov, N., Countably many solutions of a fourth order boundary value problem. Electron. J. Qual. Theory Diff. Equations 2004, no. 12, 1 - 15.

[9] Krasnosel'skii, M. A., Positive Solutions of Operator Equations. Groningen: Noordhoff 1964.

[10] Liu, Y. and Ge, W., Solvability of two-point boundary value problems for fourth-order nonlinear differential equations at resonance. Z. Anal. Anwendungen 22 (2003)(4), 977 - 989.

[11] Ma, R., Existence and uniqueness theorems for some fourth-order nonlinear boundary value problems. Int. J. Math. Math. Sci. 23 (2000)(11), $783-788$.

[12] Ma, R. and Wang, H., On the existence of positive solutions of fourth-order ordinary differential equations. Appl. Anal. 59 (1995)(1-4), 225 - 231.

[13] Yang, B., Positive solutions for the beam equation under certain boundary conditions. Electron. J. Diff. Equations 2005, no. 78, $1-8$.

[14] Yao, Q., Positive solutions for eigenvalue problems of fourth-order elastic beam equations. Appl. Math. Lett. 17 (2004), $237-243$.

Received July 20, 2005; revised November 25, 2005 